\title{
High-phase-purity zinc-blende InN on $r$-plane sapphire substrate with controlled nitridation pretreatment
}

\author{
Ching-Lien Hsiao, ${ }^{1}$ Ting-Wei Liu, ${ }^{1,4}$ Chien-Ting Wu, ${ }^{1}$ Hsu-Cheng Hsu, ${ }^{1}$ Geng-Ming Hsu, ${ }^{1}$ \\ Li-Chyong Chen, ${ }^{1, a)}$ Wen-Yu Shiao, ${ }^{2}$ C. C. Yang, ${ }^{2}$ Andreas Gällström, ${ }^{3}$ \\ Per-Olof Holtz, ${ }^{3}$ Chia-Chun Chen, ${ }^{4}$ and Kuei-Hsien Chen ${ }^{5, b)}$ \\ ${ }^{1}$ Center for Condensed Matter Sciences, National Taiwan University, Taipei 106, Taiwan \\ ${ }^{2}$ Institute of Photonics and Optoelectronics, National Taiwan University, Taipei 106, Taiwan \\ ${ }^{3}$ Department of Physics, Chemistry and Biology, Linköping, Sweden \\ ${ }^{4}$ Department of Chemistry, National Taiwan Normal University, Taipei 106, Taiwan \\ ${ }^{5}$ Institute of Atomic and Molecular Sciences, Academia Sinica, Taipei 106, Taiwan
}

(Received 27 December 2007; accepted 25 February 2008; published online 20 March 2008)

\begin{abstract}
High-phase-purity zinc-blende (zb) InN thin film has been grown by plasma-assisted molecular-beam epitaxy on $r$-plane sapphire substrate pretreated with nitridation. X-ray diffraction analysis shows that the phase of the InN films changes from wurtzite (w) $\mathrm{InN}$ to a mixture of w-InN and $\mathrm{zb}-\mathrm{InN}$, to $\mathrm{zb}-\mathrm{InN}$ with increasing nitridation time. High-resolution transmission electron microscopy reveals an ultrathin crystallized interlayer produced by substrate nitridation, which plays an important role in controlling the InN phase. Photoluminescence emission of $\mathrm{zb}$-InN measured at $20 \mathrm{~K}$ shows a peak at a very low energy, $0.636 \mathrm{eV}$, and an absorption edge at $\sim 0.62 \mathrm{eV}$ is observed at $2 \mathrm{~K}$, which is the lowest bandgap reported to date among the III-nitride semiconductors. (C) 2008 American Institute of Physics. [DOI: 10.1063/1.2898214]
\end{abstract}

III-nitride semiconductors with zinc-blende (zb) structure have attracted much attention in recent years. ${ }^{1-7}$ Since the cubic system has a higher crystallographic symmetry than the hexagonal one, it is expected to have lower phonon scattering, better electron transport properties, absent or reduced built-in spontaneous polarization, etc. ${ }^{8-10}$ Moreover, theoretical calculations have predicted that the $\mathrm{zb}-\mathrm{InN}$ will exhibit an even narrower bandgap $(0.5-0.6 \mathrm{eV})$ than wurtzite (w) InN. ${ }^{11-13}$ Recently, a revised bandgap of $\mathrm{w}-\mathrm{InN}$ at $0.65-0.70 \mathrm{eV}$ has been suggested by a number of experimental data and theoretical calculations, ${ }^{12-18}$ but the bandgap of $\mathrm{zb}-\mathrm{InN}$ is still not well established. In the past, zb-InN films have been grown on different substrates, including $\operatorname{GaAs}(001), \operatorname{InAs}(001), 3 \mathrm{C}-\mathrm{SiC}(001), r$-plane sapphire, etc., with proper buffer layers. ${ }^{1-7}$ However, in most of these studies, photoluminescence (PL) emission peak energies above $0.68 \mathrm{eV}$ were reported, similar to that of w-InN. Direct evidence from absorption spectra to support that $\mathrm{zb}-\mathrm{InN}$ has a lower bandgap than w-InN is still lacking. In this paper, we controlled the phase of InN grown on $r$-plane sapphire by tuning the substrate nitridation conditions. The phase of the InN films was analyzed by x-ray diffraction (XRD) and transmission electron microscopy (TEM). PL measured at $20 \mathrm{~K}$ shows that the zb-InN has an emission peak at $0.636 \mathrm{eV}$, which is about $45 \mathrm{meV}$ lower than that of high quality w-InN. ${ }^{14}$ Furthermore, the absorption edge measured at $2 \mathrm{~K}$ was found to be $\sim 0.62 \mathrm{eV}$, which further supports that the zb-InN should have the lowest bandgap among all III-nitride semiconductors. ${ }^{12,13}$

The method used to grow InN thin films is a plasmaassisted molecular-beam epitaxy (SVTA Model SVT-V-2). $8 \mathrm{~N}$ elemental indium was evaporated by a conventional Knudsen cell. The atomic nitrogen source was supplied by a radio-frequency (rf) plasma generator, fed with ultrahigh

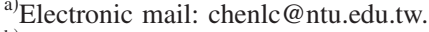

${ }^{b)}$ Electronic mail: chenkh@pub.iams.sinica.edu.tw.
}

pure $(6 N)$ nitrogen gas. Before the sample growth, the substrate was degreased using acetone, methanol, de-ionized water with ultrasonic bath, and chemically dipped into $\mathrm{H}_{2} \mathrm{SO}_{4} / \mathrm{H}_{3} \mathrm{PO}_{4}$ solution. Then, the $\mathrm{Al}_{2} \mathrm{O}_{3}(1102)$ ( $r$-plane sapphire) substrate was outgassed at $800{ }^{\circ} \mathrm{C}$ in the growth chamber. Afterward, the InN films were grown on these substrates with or without substrate nitridation. Table I shows the growth conditions of the InN thin films. Substrate nitridation was performed before $\mathrm{InN}$ growth at $200{ }^{\circ} \mathrm{C}$. Subsequently following the nitridation, the growth of InN films was carried out at $400-420{ }^{\circ} \mathrm{C}$ for $3 \mathrm{~h}$. The growth rate of the InN films is in the range of $110-120 \mathrm{~nm} / \mathrm{h}$. Figure 1 shows the $\theta / 2 \theta$-scan XRD (Bruker D8 Advance) patterns of the InN films. By increasing the initial nitridation time, the phase of the InN films changes from (a) w-InN(1011) at $33.1^{\circ}$ (sample A) to (b) a mixture of $w-I n N$ and $z b-I n N$ (sample B), to (c) $\mathrm{zb}-\operatorname{InN}(200)$ at $35.8^{\circ}$ (sample C). The lattice constant of the $\mathrm{zb}-\mathrm{InN}$ calculated from XRD is $0.501 \mathrm{~nm}$, which is consistent with previously reported values. ${ }^{1-5}$

To further understand the role of the interface after nitridation, high-resolution TEM was employed to study the interface between the film and the substrate. Figure 2(a) shows a TEM image of a 350-nm-thick zb-InN film (sample C). Some inclined stacking faults with $\sim 57^{\circ}$ to the sapphire substrate, as shown by the indicator in Fig. 2(a), appeared in the film. These stacking faults are related to the wurtzite phase with the w-InN(0001) plane stacked along the $\mathrm{zb}-\operatorname{InN}(111)$

TABLE I. Growth conditions of the InN films.

\begin{tabular}{ccccc}
\hline \hline Sample & $\begin{array}{c}\text { Nitridation } \\
\text { time (min) }\end{array}$ & $\begin{array}{c}\text { Growth } \\
\text { temperature }\left({ }^{\circ} \mathrm{C}\right)\end{array}$ & $\begin{array}{c}\text { Growth } \\
\text { time }(\mathrm{h})\end{array}$ & rf power (W) \\
\hline A & 0 & $420 \pm 20$ & 3 & 450 \\
B & 15 & $400 \pm 20$ & 3 & 450 \\
C & 30 & $400 \pm 20$ & 3 & 450 \\
\hline \hline
\end{tabular}




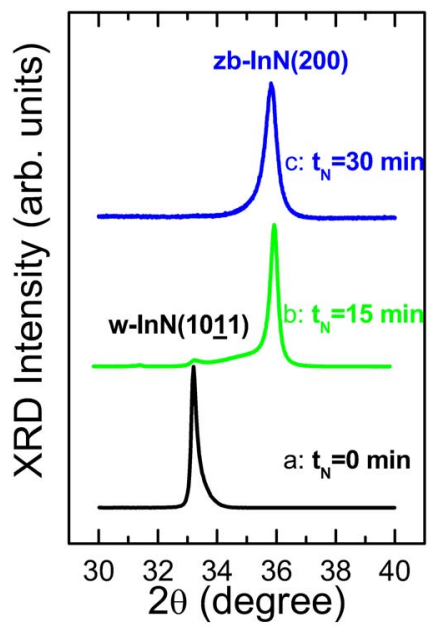

FIG. 1. (Color online) $\theta / 2 \theta$-scan XRD analyses. These InN thin films were grown on $r$-plane sapphire substrates with different substrate nitridation times $\left(t_{N}\right)$ of (a) 0 (sample A), (b) 15 (sample B), and (c) 30 min (sample C). The phase of InN changes from wurtzite to zinc blende with increasing nitridation time.

plane. ${ }^{3}$ Although the TEM image shows inclusion of some fraction of wurtzite phase, the film structure is still dominated by the zinc-blende phase. The corresponding selected area diffraction (SAED) pattern taken at the interface [Fig. 2(b)] reveals that the relationships along surface normal and in-plane orientation are $\mathrm{zb}-\mathrm{InN}(200) \| r-\mathrm{Al}_{2} \mathrm{O}_{3}(1102)$ and $\mathrm{zb}-\mathrm{InN}[011] \| r-\mathrm{Al}_{2} \mathrm{O}_{3}[0221]$, respectively. Figure 2(c) shows a high-resolution atomic image taken at the interface of sample C. An ultrathin layer $(\sim 1 \mathrm{~nm})$ appears at the interface and the $\mathrm{zb}-\mathrm{InN}$ film is stacked on top of this interlayer. Using the interplanar spacing of $d_{\langle 1102\rangle}=0.348 \mathrm{~nm}$ of the sapphire substrate as the reference scale, the lattice constant of $\mathrm{zb}-\mathrm{InN}$ is obtained as $a=0.499 \mathrm{~nm}$. This result is consistent with the lattice constant obtained from XRD analysis. Inter-

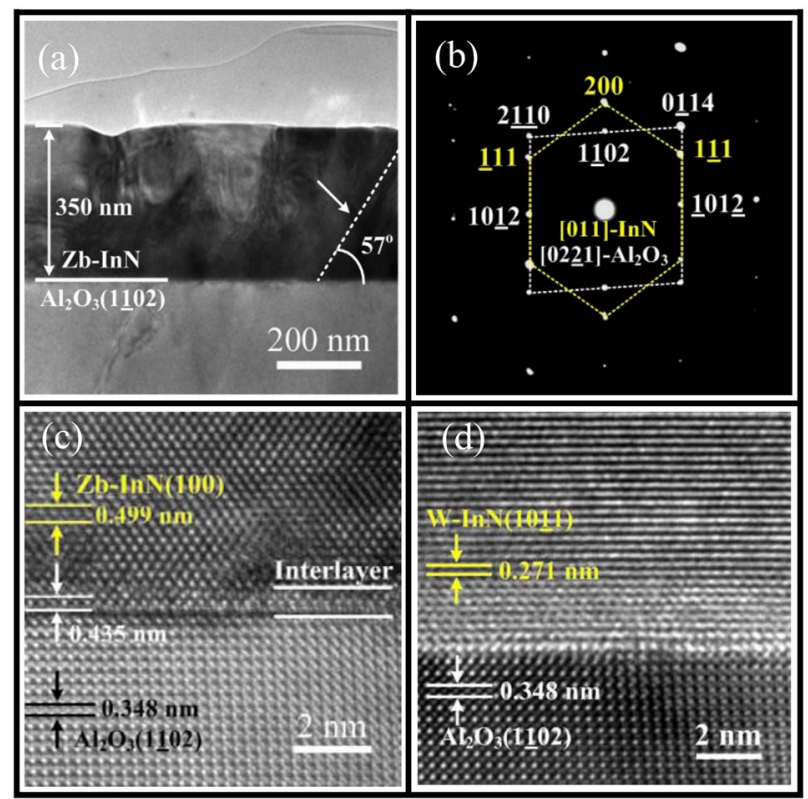

FIG. 2. (Color online) TEM images. (a) A 350-nm-thick zb-InN film grown on $r$-plane sapphire substrate (sample C). (b) Corresponding SAED pattern taken at interface shows a growth relationship of $\mathrm{zb}-\operatorname{InN}(200) \| \mathrm{Al}_{2} \mathrm{O}_{3}(1102)$. (c) High-resolution atomic image reveals an ultrathin interlayer, generated through nitridation process, between the InN film and the sapphire substrate. (d) Directly deposited InN on sapphire sub-

strate exhibits sharp interface without any interlayer (sample A). ergy and absorption edge. Besides, both W-InN and zb-InN
Downloaded 20 Feb 2009 to 140.112.113.225. Redistribution subject to AlP license or copyright; see http://apl.aip.org/apl/copyright.jsp
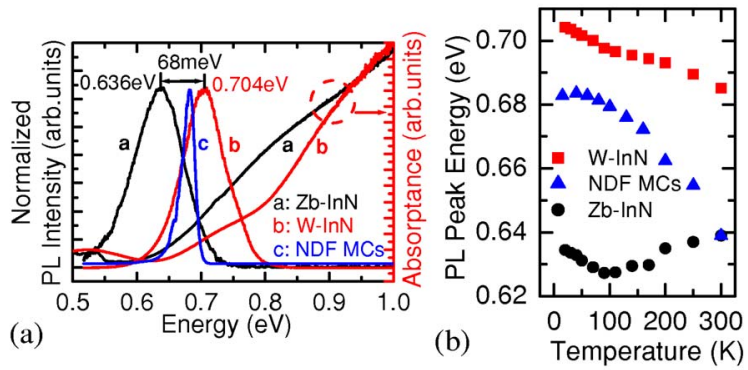

FIG. 3. (Color online) Low-temperature PL measured at $20 \mathrm{~K}$ and absorprespectively. (a)The zb-InN film shows a very low PL emission peak at $0.636 \mathrm{eV}$, which is 68 and $45 \mathrm{meV}$ lower than w-InN film and NDF MCs, respectively. The absorption spectrum of the zb-InN film also shows a rather low edge at $\sim 0.62 \mathrm{eV}$. (b) The PL peak energy vs temperature reveals that all the peak energies of $\mathrm{zb}-\mathrm{InN}$ film locate below those of $\mathrm{w}-\mathrm{InN}$ and NDF MCs.

estingly, the interlayer exhibits a similar crystalline structure to that of the zb-InN and its thickness is twice the interplanar spacing of $d_{\text {interlayer }}=0.435 \mathrm{~nm}$, which is very close to the lattice constant of zb-AlN. ${ }^{19}$ In contrast, InN film directly deposited on $r$-plane sapphire without nitridation pretreatment displays a completely different appearance, as shown in Fig. 2(d) (sample A). No interlayer was formed at the interface and the crystalline structure of the InN film shows wurtzite phase with a surface normal orientation of w-InN(1011), consistent with the XRD analysis. Formation of an $\mathrm{AlO}_{x} \mathrm{~N}_{y}(y>0.9)$ layer has been reported for sapphire substrates after sufficient nitridation, which is believed to improve the subsequent GaN film quality. ${ }^{20}$ Meanwhile, Lozano et al. have suggested that a thin indium oxide buffer layer on a $c$-plane sapphire substrate would help in mediating the growth of the high-purity $\mathrm{zb}-\mathrm{InN}$ film. ${ }^{7}$ In our case, an interlayer, presumably of $\mathrm{AlO}_{x} \mathrm{~N}_{y}$, is proposed to play an important role to promote the $\mathrm{zb}$-InN film growth on a noncubic phase substrate with rectangular surface symmetry.

Figure 3(a) shows low-temperature PL and absorption spectra of a zb-InN film, a w-InN film, and nearly defect-free microcrystals (NDF MCs) ${ }^{16}$ performed at 20 and $2 \mathrm{~K}$, respectively. A frequency-doubled $\mathrm{Nd}^{+}-\mathrm{YAG}$ (yttrium aluminum garnet) laser at $532 \mathrm{~nm}$ was used as the PL excitation source. The collected PL light was dispersed through a $0.5 \mathrm{~m}$ monochromator equipped with a $300 \mathrm{~g} / \mathrm{mm}$ grating and detected by an extended-InGaAs detector (detecting range: $0.5-1.1 \mathrm{eV}$ ). The absorption spectroscopy was performed in a BOMEM DA8 Fourier transform interferometer with a $\mathrm{CaF}_{2}$ beam splitter, a light source of quartz halogen lamp, and an InSb detector. The PL peaks of the w-InN film, NDF MCs, and the zb-InN film are located at 0.704, 0.681, and $0.636 \mathrm{eV}$ with full widths at half maximum of 80,21 , and $71 \mathrm{meV}$ (fitted by Gaussian), respectively. The absorption edge $\left(E_{\text {abs }}\right)$ of the zb-InN film is determined at $\sim 0.62 \mathrm{eV}$ by extrapolating the nearly linear region down to its baseline and the curve shows a prominent redshift as compared with the w-InN film. Although the $E_{\text {abs }}$ of the w-InN film cannot be determined precisely due to the Febry-Pérot oscillation, the $E_{\text {abs }}$ at $\sim 0.76 \mathrm{eV}$, which is extracted by a fitting curve with a sigmoidal equation (Boltzmann function), ${ }^{14}$ is estimated. The above results are remarkable because the bandgap difference between wurtzite and zinc-blende $\mathrm{InN}$ would be larger than $45 \mathrm{meV}$ based on the results of PL peak energy and absorption edge. Besides, both w-InN and zb-InN P license or copyright; see http://apl.aip.org/apl/copyright.jsp tion spectra measured at $20 \mathrm{~K}$ of zb-InN film, w-InN film, and NDF MCs, 
films have the same level of carrier densities in the range of $(1-2) \times 10^{19} \mathrm{~cm}^{-3}$ as determined in Hall measurements. If we further consider the band filling (Burstein-Moss shift) and the band tailing effects, which resulted in a large blueshift and a weak redshift of the observed PL peak and absorption edge, respectively, in degenerate semiconductors, such as high-carrier-density $\mathrm{w}$-InN,${ }^{14,21,22}$ the bandgap of the $\mathrm{zb}$-InN with zero carrier concentration would be even lower than $0.62 \mathrm{eV}$.

Figure 3(b) shows the plot of PL peak energy versus the temperature. Only the NDF MCs follow the Varshni equation well because of its high crystalline quality and low carrier concentration. An anomalous (S-shaped) temperaturedependent PL peak of the zb-InN film could be observed. This behavior have been discovered from ternary or quaternary alloy and multiple quantum wells. ${ }^{23,24}$ In order to elucidate the somewhat strange temperature dependence in our case, one has to consider the high carrier density. The high carrier density will likely give rise to the localization phenomena but cannot fully explain the temperature dependence. Also, for material with a high carrier density, a redshift is primarily expected, at least from somewhat elevated temperatures. ${ }^{25}$ Another factor taken into account is the content of a small amount of w-InN embedded in the zb-InN film. Mixture phases of wurtzite and zinc blende have been similarly observed in $\mathrm{GaN}$ film with a resulting type II semiconductor system. ${ }^{26}$ Unfortunately, for the case of InN, the band offset for the zb-InN/w-InN system has not been reported to the best of our knowledge, neither theoretically or experimentally. However, the bandgaps are fairly similar, ${ }^{2,11-17}$ which increases the probability for a type II alignment. In a type II structure, a transition from spatially indirect at lowest temperatures to spatially direct transitions at elevated temperatures could give rise to a blueshift in the PL spectra with increasing temperatures. Nevertheless, more experiments are still needed to clarify the origin of the $\mathrm{S}$-shaped behavior in zb-InN.

In summary, the growth of zb-InN films on $r$-plane sapphire is achieved by inserting an interlayer by means of a proper substrate nitridation. The orientation relationship for zb-InN film on sapphire along its surface normal is zb-InN $(200) \| r-\mathrm{Al}_{2} \mathrm{O}_{3}(1102)$ and that along the in-plane direction is $\mathrm{zb}-\mathrm{InN}[011] \| r-\mathrm{Al}_{2} \mathrm{O}_{3}[0221]$. The differences in PL peak energy and the absorption edge are 68 and $\sim 140 \mathrm{meV}$, respectively, between the w-InN and zb-InN films with similar levels of carrier densities. Furthermore, even the low-carrier-density $\mathrm{InN}$ microcrystals ${ }^{16}$ show a higher PL peak energy than the zb-InN film with somewhat higher carrier density. Our results have further supported that zinc-blende structured nitride semiconductors indeed have lower bandgaps than their wurtzite counterparts. ${ }^{12,13}$
The authors would like to thank Y. F. Chen of National Taiwan University for his fruitful discussion. This work was financially supported by Ministry of Education and National Science Council in Taiwan as well as the US AFOSR-AOARD. This work was also supported by grants from the Swedish Foundation for Strategic Research (SSF) and the Swedish Foundation for International Cooperation in Research and Higher Education (STINT).

${ }^{1}$ K. Nishida, Y. Kitamura, Y. Hijikata, H. Yaguchi, and S. Yoshida, Phys. Status Solidi B 241, 2839 (2004).

${ }^{2}$ J. Schörmann, D. J. As, K. Lischka, P. Schley, R. Goldhahn, S. F. Li, W. Löffler, M. Hetterich, and H. Kalt, Appl. Phys. Lett. 89, 261903 (2006).

${ }^{3}$ T. Nakamura, K. Lida, R. Katayama, T. Yamamoto, and K. Onabe, Phys. Status Solidi B 243, 1451 (2006).

${ }^{4}$ A. P. Lima, A. Tabata, J. R. Leite, S. Kaiser, D. Schikora, B. Schöttker, T. Frey, D. J. As, and K. Lischka, J. Cryst. Growth 201/202, 396 (1999).

${ }^{5}$ V. Cimalla, J. Pezoldt, G. Ecke, R. Kosiba, O. Ambacher, L. Spieß, G. Teichert, H. Lu, and W. J. Schaff, Appl. Phys. Lett. 83, 3468 (2003).

${ }^{6}$ Y. Kumagai, A. Tsuyuguchi, H. Naoi, T. Araki, H. Na, and Y. Nanishi, Phys. Status Solidi B 243, 1468 (2006).

${ }^{7}$ J. G. Lozano, F. M. Morales, R. García, D. González, V. Lebedev, Ch. Y. Wang, V. Cimalla, and O. Ambacher, Appl. Phys. Lett. 90, 091901 (2007).

${ }^{8}$ H. Okumura, K. Ohta, G. Feuillet, K. Balarishnan, S. Chichibu, H. Hamaguchi, P. Hacke, and S. Yoshida, J. Cryst. Growth 178, 113 (1997).

${ }^{9}$ S. Yoshida, Physica E (Amsterdam) 7, 907 (2000).

${ }^{10}$ D. N. Talwar, D. Sofranko, C. Mooney, and S. Tallo, Mater. Sci. Eng., B 90, 269 (2002).

${ }^{11}$ P. Rinke, M. Scheffler, A. Qteish, M. Winkelnkemper, D. Bimberg, and J. Neugebauer, Appl. Phys. Lett. 89, 161919 (2006).

${ }^{12}$ C. Presson and A. Ferreira da Silva, J. Cryst. Growth 305, 408 (2007).

${ }^{13}$ B. Briki, A. Zaoui, F. Boutaiba, and M. Ferhat, Appl. Phys. Lett. 91, 182105 (2007).

${ }^{14}$ J. Wu, W. Walukiewicz, W. Shan, K. M. Yu, J. W. Arger, III, E. E. Haller, S. X. Li, E. E. Haller, H. Lu, and W. J. Schaff, J. Appl. Phys. 94, 4457 (2003).

${ }^{15}$ V. Y. Davydov, A. A. Klochikhin, R. P. Seisyan, V. V. Emtsev, S. V. Ivanov, F. Bechstedt, J. Furthmüller, H. Harima, A. V. Murdryi, J. Aderhold, O. Semchinova, and J. Graul, Phys. Status Solidi B 229, R1 (2002).

${ }^{16}$ C. L. Hsiao, H. C. Hsu, L. C. Chen, C. T. Wu, C. W. Chen, M. Chen, L. W. Tu, and K. H. Chen, Appl. Phys. Lett. 91, 181912 (2007).

${ }^{17}$ S. Gwo, C. L. Wu, C. H. Shen, W. H. Chang, T. M. Hsu, J. S. Wang, and J. T. Hsu, Appl. Phys. Lett. 84, 3765 (2004).

${ }^{18}$ J. T. Chen, C. L. Hsiao, H. C. Hsu, C. T. Wu, C. L. Yeh, P. C. Wei, L. C. Chen, and K. H. Chen, J. Phys. Chem. A 111, 6755 (2007).

${ }^{19}$ M. P. Thompson, G. W. Auner, and A. R. Drews, J. Electron. Mater. 28, L17 (1999).

${ }^{20}$ G. Namkoong, W. A. Doolittle, A. S. Brown, M. Losurdo, P. Capezzuto, and G. Bruno, J. Vac. Sci. Technol. B 20, 1221 (2002).

${ }^{21}$ S. P. Fu, T. T. Chen, and Y. F. Chen, Semicond. Sci. Technol. 21, 244 (2006).

${ }^{22}$ B. Monemar, P. P. Paskov, and A. Kasic, Superlattices Microstruct. 38, 38 (2005).

${ }^{23}$ Y. H. Cho, G. H. Gainer, A. J. Fischer, J. J. Song, S. Keller, U. K. Mishra, and S. P. DenBaars, Appl. Phys. Lett. 73, 1370 (1998).

${ }^{24} \mathrm{Q}$. Li, S. J. Xu, M. H. Xie, and S. Y. Tong, J. Phys.: Condens. Matter 17, 4853 (2005).

${ }^{25}$ J. D. Sheng, Y. Makita, K. Ploog, and H. J. Quessier, J. Appl. Phys. 53, 999 (1982).

${ }^{26}$ X. H. Lu, P. Y. Yu, L. X. Zheng, S. J. Xu, M. H. Xie, and S. Y. Tong, Appl. Phys. Lett. 82, 1033 (2003). 